\title{
PARTICIPACIÓN DE NIÑOS Y JÓVENES EN LA CRIMINALIDAD ORGANIZADA EN MÉXICO
}

Participation of children and teenagers in organized crime in Mexico

Wael Sarwat Hikal Carreón ${ }^{1}$

Recibido: 15 de septiembre 2019 - Aceptado: 10 de diciembre de 2019

\section{RESUMEN}

La criminalidad organizada en México es un fenómeno que ha conducido al país a una crisis. Según el Secretariado Ejecutivo del Sistema Nacional de Seguridad Pública (2019), ocurren cerca de 90 muertes cada día, relacionadas mayormente al crimen organizado. Desde hace años el problema que enfrentan las administraciones públicas en México, es duro de derrotar, pues se han producido alrededor de 300 mil muertes en alrededor de 12 años. La participación activa de los jóvenes en el crimen organizado va en crecimiento. Factores como pobreza, marginación, falta de oportunidades, desarrollo social y humano, entre otros, vulneran aún más a los ya desprotegidos y afectan a las nuevas generaciones. Se observa que las edades de los jóvenes que se involucran en el crimen organizado oscilan entre los 18 y 30, pero también se advierte la presencia de menores de edad, encontrando niños y adolescentes en grupos criminales. El presente artículo de investigación expone un análisis de este problema desde un enfoque integral de la criminología; primeramente, desde la articulación entre esta y el estudio de la juventud y, segundo, abordando la explicación del crimen organizado. Se plantea el objetivo de analizar algunas condiciones sociales que reflejan el involucramiento de los menores en la criminalidad organizada, basado en casos periodísticos. Finalmente, se presentan soluciones desde un actuar gubernamental y cívico.

Palabras clave: Conflicto social; Criminología; Delincuencia juvenil; Malestar de la juventud.

\footnotetext{
1 Doctorando en Educación por la Facultad de Filosofía y Letras (becario CONACYT). Maestría en Trabajo Social por la Facultad de Trabajo Social y Desarrollo Humano (becario CONACYT). Licenciatura en Criminología por la Facultad de Derecho y Criminología de la Universidad Autónoma de Nuevo León. Director de proyectos en Sociedad Mexicana de Criminología capítulo Nuevo León. https://orcid.org/0000-0003-1278-567X E-mail: wshc1983.2013@ gmail.com
} 
Wael Sarwat Hikal Carreón

\section{ABSTRACT}

Organized crime in Mexico is a phenomenon that has led the country to a crisis. According to the Executive Secretariat of the National Public Security System (2019), about 90 deaths occur every day, mostly related to organized crime. For years, the problem faced by public administrations in Mexico has been hard to defeat, in around the last 12 years totalized deaths counts around 300,000. The active participation of young people in organized crime is growing. Factors such as poverty, marginalization, lack of opportunities, social and human development, among others, further violate the already unprotected as well as new generations. It is observed that the ages of the young people oscillate between 18 and 30, but also the presence of minors, finding children and adolescents in criminal groups. The present research paper exposes an analysis of this problem from a comprehensive approach to criminology; firstly, from the articulation between it and the study of youth and, secondly, addressing the explanation of organized crime. The objective is to analyze some social conditions that reflect the involvement of minors in organized crime, based on journalistic cases. Finally, solutions are presented from a governmental and civic action.

Keywords: Social conflict; Criminology; Juvenile delinquency; Discomfort of youth.

\section{INTRODUCCIÓN}

La criminología es un campo en el que convergen diversos conocimientos que han tomado en su interés de estudio el fenómeno de la criminalidad en sus diversas expresiones. Al ser diversas ciencias las que interpretan este problema, existen perspectivas variadas, que, recopiladas por la criminología, permite visualizar el campo de estudio (Velázquez, 2005, p. 1) con aproximaciones diferentes, pero integradas en un objetivo, que es el de explicar la criminalidad (Escobar Marulanda, 2006). Dada la mencionada variedad de interpretaciones sobre este objeto, da oportunidad a tener multitud de términos entre violencia, crimen, delito, psicopatía, sociopatía, agresión, desviación, conducta antisocial, entre otros, los cuales, para neutralizar su diversidad, en el presente trabajo, se hará referencia de manera general al término "criminalidad" (crimen y criminal). Por otra parte, de la generalidad de la criminología, la que se ocupa del estudio de la conducta criminal en los menores, es, entre otras, la criminología infanto juvenil, siendo desde esta, en su enfoque múltiple e integrador, sobre la cual se aborda este artículo, permitiendo ofrecer nociones antropológicas, sociales, psicológicas, jurídicas, históricas, entre otras.

El fenómeno que se pretende explicar es el de la participación de los jóvenes en actividades de crimen organizado en el contexto mexicano, los cuales han tenido una relevante y notoria presencia, lo que es preocupante por el deterioro social en el que se encuentra el país, dañando más a las juventudes y que provoca que en la actualidad, en los hechos criminales graves, estén presentes jóvenes de entre 10 y 25 años, estos se han convertido en un grupo 
vulnerable, sobre el cual, el crimen organizado ha distribuido sus brazos para reclutar y ofrecer salidas y oportunidades de vida diferentes a las que en el contexto político, económico y social actual, se pudiera tener, el cual, no es alentador para los jóvenes, por lo que una parte, se desvía para adherirse a las actividades ilícitas.

Durante el artículo, se desarrollan nociones de la criminología infantil y juvenil para conocer el tipo de abordaje integral que esta realiza, en esta misma dirección, se muestran conceptos jurídicos del crimen organizado, basado en la legislación mexicana federal, pero también en la conceptualización internacional, por otro lado, se dan explicaciones interpretativas desde la óptica psiquiátrica y psicológica, así como la percepción sociológica. En otro momento, se recurre a la recopilación y estudio periodístico para ejemplificar con casos reales sobre el involucramiento de los jóvenes en hechos criminales. Finalmente, se acude a la visión holística interdisciplinaria para ofrecer opciones en la atención y prevención de este problema.

\section{Criminología infanto-juvenil, juventud y crimen organizado}

La criminología infanto-juvenil tiene en su interés de estudio el comportamiento de los menores desde el nacimiento hasta la adolescencia. Pero sobre todo se interesa en el momento y orden de aparición de problemas que dan lugar a las conductas antisociales. De esta manera, los criminólogos intentarán explicar las semejanzas y las diferencias entre los niños, así como su comportamiento y desarrollo, tanto normal como anormal. El objetivo de conocer desde momentos tempranos es para detectar si ha sido un niño procreado en determinadas condiciones que pudieran conducir desvío de su conducta, o si su estilo de vida pudiera peligrar su desarrollo, ya sea por el contexto familiar o social en el que se encuentra. Sobre la base de estas detecciones, se puede prevenir la repercusión de los efectos que pudiera tener. Esto puede ser visto a nivel individual o colectivo, siendo la infancia y las juventudes, las más vulnerables de padecer riesgos en su desarrollo.

La conceptualización de la niñez, adolescencia y juventud, está determinada por la sociedad, donde se involucran aspectos culturales, geográficos, ambientales, históricos, entre otros (Dávila León, 2005). La noción de estos términos tiene mayor arraigo desde la psicología, que mira los procesos de madurez emocional y la interacción de estos con la sociedad, estudiando además sus procesos culturales y adaptación, pero cuando se refiere a lo que corresponde estudiar en el presente escrito sobre su participación en la criminalidad organizada, es interpretado más desde una mirada sociológica, analizada como producto de la serie de factores culturales, familiares, económicos, escolares y de interacción con otras personas. Tomando la adolescencia desde la psicología como un momento crítico en el que ocurren cambios significativos y que implica un estado de crisis, donde se padece sobre la 
Wael Sarwat Hikal Carreón

ruta que la vida debe tomar, generación de metas, descontento con el entorno, consigo mismo, insatisfacción, agresividad, etcétera (Hernández Moreno, 2011).

La Organización Mundial de la Salud (2020) se refiere al periodo de la adolescencia como un momento de desarrollo del humano, que comprende entre los 10 y 19, cuyas características son el acelerado crecimiento y cambios, los cuales, marcan el rumbo al futuro en su vida (Dávila León, 2005). Respecto la niñez, esta comprende un periodo de desarrollo motriz, emocional, crecimiento físico y establecimiento de rasgos comportamentales y de pensamiento, determinados por los agentes que le formen (Calderón Carrillo, 2015). Durante el desarrollo de los niños y adolescentes, intervienen primeramente la familia como primer agente socializador y desarrollador de este nuevo humano en crecimiento, posteriormente, el segundo agente es la escuela, otros agentes pueden ser las amistades u otros contactos que el niño/joven establezca (Jaramillo, 2007), estos últimos, siendo de relevante interés cuando ocurre algún hecho criminal, pero también, es invitación obligada a mirar la dinámica familiar. En ambos periodos, es un proceso de dependencia hacia otras personas, donde los niños jóvenes, son receptores de actitudes, palabras, condiciones, ambientes, influencias familiares, o de amistades, incluso de medios de comunicación, todos estos, son referentes de orientación y construcción de su propia realidad interna, así como la externa que estos construyen, interpretan o visualizan para sí mismos, ya sea de maneras positiva o negativa (Lansdown, 2005).

En este apartado, no se abundará en el concepto jurídico de niñez, puesto que de manera abreviada, se considera que es niño aquel que está en un rango menor a los 12 años, y por adolescente, el que está entre los 12 y 18 años (Comisión Nacional de los Derechos Humanos, 2018), estos rangos comprenden la minoría de edad, concepción aceptada y sugerida incluso a nivel internacional (UNICEF Comité Español, 2006), mientras que la mayoría de edad, en México, comienza a los 18 años, esto quiere decir, que es sujeto de más derechos, adquiere obligaciones y responsabilidades (para las cuales tal vez no está capacitado). El periodo de juventud comprende de los 18 a los 29 (Castillejos Cifuentes, 2011).

El crimen organizado ha sido tan relevante en México, que la norma que le atiende es federal, así del Código Penal Federal, se creó una ley especial en 1996 para tratar este tema, así, es considerado según la Ley Federal contra la Delincuencia Organizada en el artículo $2^{\circ}$ "Cuando tres o más personas se organicen de hecho para realizar, en forma permanente o reiterada, conductas que por sí o unidas a otras, tienen como fin o resultado cometer alguno o algunos de los delitos siguientes" (Cámara de Diputados, 2019, p. 1), a los delitos que comprenden, son: Terrorismo, tráfico de armas y acopio, tráfico de órganos, corrupción de menores, trata de personas, secuestro, contrabando, defraudación fiscal, y 
contra el ambiente. En el artículo 5 de la misma ley señala el aumento de penas cuando sean participes servidores públicos o cuando se auxilien de menores de edad (Cámara de Diputados, 2019, p. 5). La Oficina de las Naciones Unidas contra la Droga y el Delito especifica en la Convención de las Naciones Unidas contra el Crimen Organizado Transnacional que un grupo criminal organizado es: "Un grupo de tres o más personas que no fue formado de manera aleatoria; Que ha existido por un periodo de tiempo; Actuando de manera premeditada con el objetivo de cometer un delito punible con, al menos, 4 años de encarcelamiento; Con el fin de obtener, directa o indirectamente, un beneficio financiero o material" (Oficina de las Naciones Unidas contra la Droga y el Delito, 2020).

\section{Método}

Para el desarrollo del presente se recurrió al método de revisión bibliográfica, la cual consiste en la búsqueda de información a través de documentos para recopilar información sobre el tema específico; es decir, búsqueda de un tema adecuado. Se recurrió a artículos para obtener conceptos, términos objeto de estudio del presente, e información relacionada con los factores de riesgo. Se buscaron cifras estadísticas en documentos de instituciones nacionales e internacionales. También se recurre al análisis periodístico, previa identificación del tema en concreto, así como clasificación y discriminación de los medios, para obtener de estas notas, información de calidad, vista desde la observación directa, entrevista a expertos, datos estadísticos obtenidos de instituciones confiables, historia de vidas, y revisión de datos existentes. Lo anterior enfocado a la parte dedicada a los jóvenes participes en actividades del crimen organizado. También de esta fuente de información, se obtienen aportes sobre los factores de riesgo.

\section{Edad y conductas criminales en los niños y jóvenes}

Los menores de edad dependen del cuidado de los padres porque no miden las consecuencias de los actos que realicen, podría ser fácil cuestionar qué daño puede hacer un niño o adolescente, pero esta interrogante ha venido siendo respondida con actos crueles donde están involucrados los “jóvenes” (Martínez Gonzáles, Robles Haydar, Utria Utria y Amar Amar, 2014). En la edad más productiva de la vida, los jóvenes se involucran en actividades también ilícitas, por ello que sea visto este proceso como uno de los más vulnerables donde hay que poner más atención y evitar se desvíen de la expectativa de la sociedad (Martínez Corona, Méndez Cadena y Pérez Nasser, 2014).

En esa época de la vida donde la distinción entre realidad y la fantasía propia del menor o la fomentada en medios de comunicación, mezcladas y con las inquietudes internas del adolescente, necesidades, aspiraciones, confusiones, entre otras apariciones de la vida que le resultan tal vez difícil de comprender y manejar, llevan a un estado de inestabilidad y crisis que involucra a su propia personas y familiares, comenzando un reto de medir fuerzas 
Wael Sarwat Hikal Carreón

que equilibren conductas, preferentemente a través del fomento a la concientización en algunos casos, pero en otros, se vuelve una lucha y disputas (Horacio Luis, 2015). Pareciera común que entre la niñez y adolescencia, el juego entre iguales lleva una fuerte carga de fantasías y creatividad (Suárez Beltrán, 2009), producto natural del mismo ser, así como del trasmitido en medios de comunicación, haciendo una mezcla de la realidad con aquella fantasía, misma que en momentos, desearían que fuese parte de su realidad, es la imaginación de estos, la que construye su realidad con enfoque de la cultura propia y la observada en otros (Alessandroni, 2017).

Aquellos brotes de creatividad y fantasía, pueden llevar a que mientras se encuentren jugando con alguna herramienta, el niño no pensará en el daño físico o mental que causará porque a su edad no tiene noción de lo que hace, ni la experiencia de consecuencias (Bayo Margalef, 1987). Lo anterior se puede ejemplificar en el caso "Teníamos ganas de matar a Christopher" (Pérez-Stadelmann, 2015), la historia de unos chicos que jugaron a ser sicarios, secuestrando a un niño, torturándolo y matándolo. Nuestro comportamiento es en parte resultado de imitaciones, el juego brusco en menores puede ser resultado de caricaturas que tengan contenidos directamente violentos o la violencia como parte de un juego, el perseguirse, atraparse, jugar a matar al otro, a comérselo, son características presentes en programas, caricaturas, videojuegos, y actividades lúdicas entre menores (Ochoa Rojas, 2007). También, es frecuente que los menores jueguen a darse golpes que pudiera ocasionar daños sin pensar en sus resultados, por ello que los padres deben estar al cuidado de los menores y aproximarlos a las realidades. El menor realiza actividades sin tener conciencia de la realidad, se le enseña a que puede diferenciar entre lo bueno y lo malo, pero no sabe de las consecuencias que le traerán las acciones que realiza, aunque esto no es exclusivamente de los menores, la ignorancia de la ley lleva a cometer muchos daños sin saber sus consecuencias (Manrique, 2013).

La participación de los menores en conductas criminales con consecuencias graves ha sido tal que el Estado (Sánchez Galindo, 2017) se vio en necesidad de crear centros de reclusión para estos (Bayón Guareño, 2005), y que ha llevado un alza estadísticamente, “en México son los más jóvenes quienes están cometiendo la mayoría de los delitos y, en consecuencia, quienes están poblando las prisiones" (México SOS, s.f.). Según el último censo (2016) realizado por el Instituto Nacional de Estadística y Geografía, en México, en el año 2015, ingresaron 7,785 adolescentes a los sistemas carcelarios de las entidades federativas, por delitos de orden común; es decir, no de orden federal, como lo es el crimen organizado, de lo cual, según un estudio realizado en 2019 por la Comisión Nacional de los Derechos Humanos y la Universidad Nacional Autónoma de México, indicó: "no existen estadísticas oficiales sobre el número y el tipo de delitos cometidos por los niños y las niñas 
involucrados en el crimen organizado. Cifras de la academia hablan de unos 30,000 niños, niñas y adolescentes que cooperan con los grupos criminales de varias formas" (p. 177). Dependiendo de la edad puede ser la tendencia hacia la conducta antisocial, aunque hay menores que cometen actos verdaderamente sorprendentes, extraños y violentos, demostrando un alto grado de peligrosidad (Cornellá i Canals y Llusent i Guillamet, s.f.). Cuando el individuo está en una edad mayor, que es consciente de lo bueno y lo malo, aumenta su peligrosidad si decide actuar en contra de la ley, ya que los actos que realiza son voluntarios y consientes. Aunque a consideración, son actos que tienen como base desórdenes psíquicos y sociales que llevan a actuar de cierta manera y desarrollar un rechazo a la sociedad y a las personas, con notaria falta de empatía (Conde Alvarado, 2015).

Dentro de los factores que, como consecuencia tienden a influir la conducta antisocial del menor, se encuentran la violencia familiar, la misma desintegración negativa de la familia, el medio ambiente, la condición económica, el abandono, falta de acceso a educación, poca expectativa positiva al futuro, condiciones de vivienda inadecuada, dificultad al acceso a servicios, entre otras (Senado de la República, 2014). Es mencionado que la desintegración familiar negativa y las condiciones sociales, son motivos fuertes por el que los menores se convierten en infractores de la ley, lo que puede ser desde un ladrón hasta un homicida. Factores como desatender a los menores por problemas familiares como la violencia o la separación de los padres, puede provocar que estos vayan buscando refugio en el alcohol, drogas o compañía de personas que los conllevan a actos antisociales (Martínez Gonzáles, Robles Haydar, Utria Utria y Amar Amar, 2014).

El comportamiento antisocial comienza a menudo en los primeros años de la adolescencia con pequeños robos, lesiones, peleas, escaparse de obligaciones, evitar responsabilidades. En gran medida, estas infracciones son de forma ocasional; es decir, que rara vez es premeditada y que puede surgir del deseo de divertirse con los amigos o para obtener algunos bienes materiales (Cornellá i Canals y Llusent i Guillamet, s.f.). En algunos puede que sea un momento temporal, pero otros van más allá y cometen infracciones más graves, o lo adoptan como un estilo de vida, por lo que se convierte en un patrón (American Psychiatric Association, 2014). Estos a menudo han pertenecido a grupos antisociales y, a través de un proceso de socialización, se han habituado a un modo de vida antisocial. El problema en la adolescencia es el de una adaptación a los medios en los que se desenvuelve, medios que muchas veces lo llevan a adquirir conductas violentas y a la vez lo llevan al consumo de alcohol u otras drogas como medio de rebeldía o descarga (Becoña Iglesias, 2000). Con la madurez física, el adolescente va notando sus capacidades para desvelarse, aguantar muchas horas de ocio, entre otras conductas que, a largo plazo, son dañinas. Una fórmula que permite entender el curso desfavorable de la conducta en los jóvenes es: Agresión + rechazo social $=$ conducta desviada. Teniendo como base las observaciones 
Wael Sarwat Hikal Carreón

anteriores, se puede dar cuenta de la conducta desviada de un adolescente, la cual deberá de atenderse lo antes posible, para evitar posteriores deformaciones en la personalidad que conduzcan a un trastorno antisocial de la personalidad (American Psychiatric Association, 2014).

El adolescente tiene que enfrentar la adaptación al medio que lo rodea, y muchas veces no lo logra, manifiesta un rechazo a todas las normas de control y se vuelve agresivo contra su familia, religión y sociedad. El adolescente al sentirse incomprendido, se refugia en sus amigos, y sus amigos en la misma situación se refugian en otras actividades con las cuales se sienten cómodos o prometen ser mejores que en las condiciones en las que están agobiados. "Los jóvenes hoy no creen en nada o en casi muy poco, en cosas desechables que surgen un día y para el atardecer ya son obsoletas, no tienen sentido, de ahí que sean presas fáciles (clientes frecuentes) del mercado el cual plantea solo gozar y gozar" (Ramírez Garza, 2009). El adolescente entra en un período en el que la competencia por demostrar quién es el más fuerte o quién es el que tiene más, hace que actúe sin pensar, muestran una exagerada rebeldía, volubilidad emocional y extrañeza por no sentirse cómodos con su ambiente. Se deben a debilidades mentales y a inclinaciones por lo que otras personas dicen.

Para imaginar los problemas de un adolescente hay que observar su modo en el que se desarrolla, que lo envuelve, y además, el medio principal que es el familiar. Es muy común que los hijos no tengan relación durante el día con sus padres, y también es común que los hijos en su mayoría no conozcan a éstos. El tiempo que el adolescente pasa fuera de su hogar, es el tiempo en el que va formando su actitud hacia el futuro, las alteraciones que se den en esa etapa son las que marcarán la vida adulta del sujeto. Problemas hoy propios de la adolescencia e inicios de ésta son: el pandillerismo, el bullying (comúnmente relacionado con los planteles escolares), el cyberbullying (subir videos a YouTube u otros donde se muestran las riñas o el acoso electrónico), y más gravemente su ingreso al crimen organizado, donde las cosas son de poder, satisfacción y placeres. Cuanto más se permita persistir en este tipo de conductas antisociales, más difícil será detenerlas mediante medidas sociales o de intervención con fines preventivos. Por ello, resulta decisivo impedir lo antes posible que los niños y adolescentes se dejen atraer por conductas antisociales que, al ser mayores de edad se convierten en criminalidad.

\section{Extensión a las juventudes del crimen organizado en México}

En México existe un entorno de violencia que se ha ido fabricando socialmente desde hace años, con la suma de factores múltiples como las condiciones escolares deficientes, dificultad de acceso a diversos servicios públicos, como a los centros escolares, salud, agua, luz, limpieza en calle, pavimentación, alumbrado público, parques para deporte, recreación, 
lejanía de las viviendas, transporte público tardío o de difícil acceso, horarios extensos por parte de los padres en sus obligaciones laborales, salarios bajos, que no facilitan la distribución de la economía, entre tantos problemas que laceran tanto a la población en general, pero sobre todo a los jóvenes en su desarrollo (México SOS, s.f.). Para cualquier individuo las dificultades para acercarse a servicios satisfactorios, y actividades productivas, le hunden en situaciones de ansiedad, frustración, disgusto, agresividad y violencia (Mustaca, 2018). Estas situaciones son focos de alerta, que se tienen identificados, pero difícilmente tratados con resultados positivos visibles en la sociedad, y no maquillados en cifras de programas políticos.

En México, tomando como referencia tres administraciones gubernamentales federales (presidentes: Felipe de Jesús Calderón Hinojosa, 2006-2012, Enrique Peña Nieto, 20122018, y Andrés Manuel López Obrador, 2019-actual), como cifra oficial, suman alrededor de 300,000 en parte la suma se debe a su relación con el crimen organizado (Lara Bermúdez, 2018; La Redacción 2013; Zuckerman, 2019); es decir, cercana cantidad de población que existe por ejemplo, en Islandia, que, para julio de 2019, eran 360,390 habitantes (Statistics Icelanda, 2019), siendo este el país considerado como el más pacífico del mundo según el Índice de Paz Mundial 2019 (Institute for Economics \& Peace, 2018); es decir, en México, se ha matado a casi la misma población que un país. En su momento, el sexenio de Felipe Calderón, se hubiese considerado como el más violento de los últimos 50 años de México, además de una llegada a la presidencia de manera cuestionada y turbia (Ortega Ortíz y Somuani Ventura, 2015), esta calificación de violencia se da a razón de emprender una guerra contra el crimen organizado sin tener las condiciones policiales, sociales ni militares, adecuadas para ello, a la par de los decesos, el deterioro social se agravaba, lo que conducía a una reproducción de criminales unidos al crimen organizado, situación que al cambio de presidencia nacional, parecía disminuirá el problema, pero al cabo de poco tiempo (Bizberg, 2015), se mantuvo y agravó en modalidad de expresión de la criminalidad organizada, así como en cantidad de muertes.

Las actividades del crimen organizado en México se han destacado principalmente por el tráfico de drogas, cobro de piso, secuestros, extorsiones, tráfico de personas, prostitución forzada, entre otras. Y aunque el mero término pudiera parecer cotidiano, las formas de expresión que se han dado, pasaron de ser sorprendentes y desrealizantes, a perder la sorpresa y asombro, en México, antropológicamente hubo la necesidad inconsciente y colectiva de acostumbramiento a los hechos de crimen organizado (Martínez Pacheco, 2016), ya no es extraordinario saber de enfrentamientos entre bandas rivales o fuerzas públicas entre estos mismos, o entre el grupo criminal contra la fuerza pública, la corrupción de las policías municipales, estatales y federales, entre los militares y marinos, así como los servidores públicos gobernantes, ya no es ausente en presencia mediática, ni 
Wael Sarwat Hikal Carreón

en la realidad de los hechos, incontables actos donde estos han estado involucrados, México, viene a ser el país donde todo puede pasar. Por otra parte, las modalidades: Balaceras a cualquier hora del día, en diferentes localidades, emboscamientos, bolsas con partes de cuerpos, incluso los cuerpos abandonados en cualquier sitio público, vehículos incendiados, carreteras bloqueadas, cuerpos sin vida colgados de puentes peatonales y viales, videos de torturas, cuerpos desechos en ácido, entambados, chats entre aficionados al crimen o por estos mismos, entre más sucesos, no son ya sorpresa (Mendoza García, 2011). Lo que actualmente es sorprendente, es la participación de menores de edad en todos estos actos mencionados, preocupa por la precocidad, crueldad, y deterioro social, así como lo atractivo que se ha vuelto el crimen para las juventudes, en lugar de optar por estilos de vida lícitos.

\section{Análisis periodístico de casos de niños y jóvenes involucrados en el crimen organizado}

En las siguientes líneas se listan algunas notas periodísticas relevantes para analizar, no pueden recopilarse todas, dado que los hechos sobrepasan los límites de este trabajo, lo que ocupa cientos de páginas de los periódicos locales. Se mencionan algunos hechos para resaltar la magnitud del problema de los jóvenes en las actividades del crimen organizado, lo que ha trascendido de crímenes cotidianos como robo, lesiones, a ser sicarios, realizar operaciones de vigilancia, descuartizamiento, extorsiones, entre otros. Se recurre a fuentes periodísticas digitales serias, con arraigo y presencia a nivel nacional en México, descartando notas de presencia alarmista o conjugación de crimen con erotismo o fútbol, atendiendo a medios con columnistas reconocidos por su trabajo de investigación, análisis, debate público y recopilación metódica. En relación a la cifra de muertos mencionados en el apartado anterior, a pesar de haber sostenido una guerra directa contra los integrantes de grupos criminales, estos mismos se incrementaban y no llegaba a un fin el problema de los grupos, si se desmenuza la problemática social de México, al ser muchas las causas (Romero, 2004) de pobreza, desigualdad, miseria, corrupción, entre otras, las juventudes desprotegidas, son la siguiente fila de escudo y acción para las bandas criminales, aprovechándose de su vulnerabilidad, para adoptarlos y que trabajen de manera voluntaria o forzada, en contraste, con políticas públicas y programas que al corto plazo no remiendan las situaciones de riesgo, el crimen organizado se vuelve atractivo para muchos.

La siguiente nota publicada por periódico Excelsior, se presenta bajo el título de "Adolescentes, los sicarios desechables del Narco", por Flores Martínez (2019) presenta cifras estadísticas extraídas del Instituto Nacional de Estadística y Geográfica, para señalar la población de menores que habitan en México (alrededor de 40 millones), de los cuales, el mismo instituto censó que de estos algunos son criados solo por su madre o por abuelos, resaltando que las causas de su involucramiento en la criminalidad es la falta de apego, desintegración familiar, falta de oportunidades, entre otros, por lo que al estar desprotegidos 
y sin expectativas al futuro, el crimen organizado los acoge ofreciendo modos de vida excitantes, acelerados, atención, dinero, y poder, pero de modo desechable, puesto que al ser población vulnerable, son fáciles de acceder, cumplir con ciertas funciones, y por su inexperiencia en la vida, ser víctimas de asesinato. En entrevista que realiza a Juan Martín Pérez García, de la Red por los Derechos de la Infancia, apunta: "Los grupos criminales no están pensando formar criminales profesionales, los usan porque están en una condición de desprotección, de vulnerabilidad y para ellos son desechables de tal suerte que si los detienen o los asesinan tienen otros miles para disponer usarlos como tal" (Flores Martínez, 2019). " 8 de cada 10 víctimas de homicidio menores de 18 años fueron asesinadas con arma de fuego, esto quiere decir que fueron ejecutados o murieron en un enfrentamiento" (Flores Martínez, 2019) según el Secretariado Ejecutivo del Sistema Nacional de Seguridad Pública.

Otra nota titulada como "Joselyn, "la niña sicaria" que apareció descuartizada en una hielera", publicada en el periódico Infobase, el 6 de septiembre de 2019, se refiere a una joven que formaba parte de un cártel de mujeres que pretendían competir con otros carteles y que operaban en la zona norte del país (Infobase, 2019). La siguiente nota "Asesinaron a presunto sicario en Rio Verde: tenía antecedentes criminales desde los 10 años", del mismo periódico, relata la vida de un joven encontrado muerto a sus 20 años, pero con historial criminal desde los 10 años, la misma nota menciona una cifra tentativa de 30,000 niños reclutados por el crimen organizado, y que se corrobora por un estudio de la Organización de los Estados Americanos (2015, p. 71). También refiere a "La peque sicaria", una joven encargada de decapitaciones en un grupo criminal, la cual, previo a esto, se prostituía y tuvo relación con el tráfico de drogas (Infobase, 2019). Otro caso es el titulado como “"Juanito Pistolas": el sicario de 16 años que tenía su propio rap y murió decapitado en Nuevo Laredo", quien era un joven sicario perteneciente a la Tropa del Infierno, que operaba al norte del país. La nota "Niño "narco", que tiene de mascota a un tigre, amenazó a rivales del "Mayo" Zambada", hace mención a un video que circulaba en redes, de este niño amenazando a otro grupo rival, portando arma y emitiendo un discurso de odio, intimidación (Infobase, 2019).

En la nota del periódico Informador, titulada "Adolescentes sicarios revelan que los atraen vida de lujos y narcocultura", que se deriva de una investigación realizada por Barragán Bórquez (2018), donde luego de entrevistas directas a jóvenes recluidos, revela que el interés de los adolescentes por pertenecer a los grupos de crimen organizado, es motivo de acceder a los lujos (Informador, 2019). Tal motivo así sea por corto plazo, supera los estándares de vida que se tienen en la sociedad mexicana, ante el panorama desolador de falta de oportunidades, la vida exprés de lujos, resulta parecer más satisfactoria, que vivir a largo plazo en miseria y hambruna. Según el periódico Milenio, en la nota "En las filas 
Wael Sarwat Hikal Carreón

del crimen, al menos 30 mil niños" (Rivera, 2017), las bandas de grupos criminales reclutan a los menores de entre 9 y 12 años, en actividades de tráfico de drogas, espiar las actividades de las fuerzas públicas, o de grupos contrarios, posteriormente, alrededor de los 15, les encomiendan labores de ejecución, enfrentamientos armados, incluso descuartizamientos, secuestros, etcétera, por lo que no es de extrañar ya, encontrar a menores en retenes ilegales, tomando por la fuerza transportes públicos para bloquear avenidas, quemar llantas, disparos al aire, grabar sus actos, subirlos a redes (en México existe el blog titulado El Blog del Narco, donde se suben videos de torturas, interrogatorios, descuartizamientos, entre otros).

Otra nota enmarca la cantidad de niños reclutados, "Inegi: en el país, 5 mil 888 niños bajo proceso o con sentencia" (Job, 2019), señala la vulnerabilidad de los niños al ser más fáciles de reclutar dado el abandono y falta de oportunidades, además de que la legislación mexicana en materia de menores, no permite sentencias a largo plazo, por lo que, de ser encarcelados, en menos de 5 años quedan libres, y comúnmente, retornan a las actividades ilícitas. En el artículo 109 de la Ley Nacional del Sistema Integral de Justicia Penal para Adolescentes (Cámara de Diputados, 2016) establece que la prescripción de la pena se divide en tres momentos según la edad, estableciendo que de entre 1 a máximo 5 años; es decir, no puede exceder de este tiempo su sanción, apunta: "Lo previsto en las fracciones anteriores aplicará para las conductas cometidas por las personas adolescentes de conformidad con la presente Ley. En los demás casos, la prescripción será de un año" (Cámara de Diputados, 2016, p. 35), en otro párrafo del mismo artículo se refiere a la gama de delitos sexuales, donde "el plazo de prescripción empezará a correr cuando la víctima cumpla dieciocho años” (Cámara de Diputados, 2016, p. 35). No es el caso castigar por imponer, lo ideal sería que se mejoren las causas ya mencionadas y realizar modificaciones estructurales en las ciudades, así como potencializar el alcance e influencia de los programas sociales, para mejorar las condiciones de vida, de tal modo, otro resultado sería (Romero, 2004). Continuando con la nota, señala que a los criminales de más experiencia o los líderes, eligen a los jóvenes porque se deslumbran y manipulan con facilidad, además de por la capacidad física, el crimen organizado ofrece riquezas y beneficios, que una vida lícitamente llevada, no daría a corto plazo, incluso ni a largo plazo (Job, 2019; Torres Castro, 2005).

Son incontables los casos que se presentan en México, casos al extremo del terror (Puértolas, 2019), hoy, no importa la edad, horario, lugar, autoridad, el crimen organizado está muy penetrado en el país, sumado a las condiciones de deterioro que agravan la búsqueda de soluciones efectivas a corto plazo. Pero a pesar de la gravedad de los hechos, desde diversos ámbitos, debe fluir la colaboración para disminuir el impacto en las consecuencias sociales de la criminalidad, y que no siga afectando a los menores. En el siguiente apartado, el último, se plantean algunas estrategias que se pudieran poner en 
acción desde la óptica cívica y académica. Cuanto más se permita persistir en este tipo de conductas antisociales, más difícil será detenerlas mediante medidas sociales o de intervención con fines preventivos. Por ello, resulta decisivo impedir lo antes posible que los niños y adolescentes se dejen atraer por conductas antisociales que, al ser mayores de edad se convierten en delincuentes insalvables.

\section{Abordaje social a las juventudes para la prevención del crimen}

Muchas líneas se han escrito sobre los programas sociales de los gobiernos locales y nacional para dar atención a los problemas en las juventudes, niños y niñas, pero no se distingue un mejoramiento en las condiciones, por el contrario, aumentan las estadísticas y el deterioro es más visible, mismo que parece se viene acumulando. La atención en este documento se centra en la identificación de los factores de riesgo que se diagnostiquen en las localidades, sobre las características demográficas y formas de violencia (Romero, 2004), se debe trabajar en tales causas a modo de revertir la tendencia al riesgo; es decir, aquellos factores que estimulan a la criminalidad, con base a estos mismos se puede trabajar, reconstruyendo aquellas situaciones olvidadas, obsoletas y nuevas que la sociedad necesite; es decir: "La protección del bienestar, el desarrollo, los derechos y los intereses de todos los jóvenes" (Oficina de las Naciones Unidas contra la Droga y el Delito, 2007, p. 79). De acuerdo con las Directrices de las Naciones Unidas para la Prevención de la Delincuencia Juvenil, postula: "La creación de oportunidades, en particular educativas, para atender a las diversas necesidades de los jóvenes y servir de marco de apoyo para velar por el desarrollo personal de todos los jóvenes, en particular de aquellos que están patentemente en peligro o en situación de riesgo social y necesitan cuidado y protección especiales" (Oficina de las Naciones Unidas contra la Droga y el Delito, 2007, pp. 78 y 79).

Para diagnosticar qué es lo que requiere la población, se pueden establecer mecanismos de consulta a través de reuniones de consulta sobre necesidades en las localidades, intercambiar experiencias, discutir políticas que se tienen para atender las problemáticas, si estas existen, si llegan a la población necesitada, involucrar a todos los agentes institucionales y culturales para que participen en los cambios (Oficina de las Naciones Unidas contra la Droga y el Delito, 2007). Realizar talleres de detección de problemáticas en las comunidades, incluyendo a los ciudadanos, para juntos diseñar las estrategias para evitar, prevenir y controlar los riesgos, diseñar los planes de operación mediante mecanismos de participación ciudadana, que, a modo cuantitativo, se puede lograr más con una población informada, involucrada y comprometida con el cambio (Torres Castro, 2005). Esto extiende las oportunidades de colaboración compartida con mejores alcances; es decir: "Tanto el Estado como la sociedad deben trabajar mancomunadamente en apoyo a los jóvenes" (México SOS, s.f.). Los cambios a nivel social tienen que ser significativos y a plazo inmediato, mejorando las condiciones deterioradas que se han olvidado, así como 
Wael Sarwat Hikal Carreón

recuperando espacios, manteniendo los programas que hayan funcionado y estableciendo nuevos de acuerdo a los cambios y necesidades de la sociedad contemporánea.

Elemento importante es la educación, es necesario ampliar la cantidad de centros escolares con educación de calidad, en buenas condiciones de infraestructura al interior y exterior, evitando que la educación se vea maltratada por ausencia de docentes, laboratorios, equipos tecnológicos, y básicos para las funciones de aprendizaje, estimulando la cultura cívica para generar factores de protección de las violencias, concientizar que los resultados del estudio son de mayor beneficio a nivel personal, familiar y social a largo plazo. Que la educación sea el reflejo también de buenas oportunidades de desarrollo y empleos satisfactorios, para contrarrestar la percepción y tentación de que las actividades criminales a corto plazo, dejan mejores resultados (Romero, 2004), las juventudes se ven agobiadas por el panorama incierto, lo que vulnera su interés por la permanencia en la escuela o empleos temporales que no permiten la adquisición de bienes materiales básicos para sobrevivir. Programas atractivos de becas para estudios y manutención, permitirán que los menores se liberen de problemas emocionales laborales o familiares y se ocupen enteramente de su capacitación (Torres Castro, 2005).

Sin lugar a dudas, la educación y empleos bien remunerados pueden impedir que los jóvenes desvíen la mirada hacia ámbitos de actividad que les permitan encontrar en su entorno inmediato: empleo, dinero fácil y el ansiado ascenso económico. Condiciones que tiene a raudales la delincuencia organizada, llámese ésta narcotráfico, robo, secuestro, extorsión, etcétera, y que suelen seducir y satisfacer las expectativas de muchos jóvenes sin alternativas (México, SOS, s.f.).

La población y los jóvenes deben sentir apoyo y respaldo que brinde sensación de seguridad y certidumbre al futuro, las oportunidades de desarrollo deben contribuir a eliminar la tentación que un estilo de vida basado en lujos y dinero exprés, provoca, así como el pesimismo por condiciones desfavorables, y que estas sean catapulta a el automejoramiento. "Adherimos a la idea que sostiene que, para implementar cualquier articulación respecto de menores, deberán tenerse presentes (...) planos o dimensiones: la condición jurídica, la realidad biopsicosocial y el diseño instrumental que torne eficaz tal intervención" (Raffo, 2000, p. 68). La prevención de las conductas antisociales en los menores consiste en el mejoramiento de las condiciones ambientales, elevando la atención y la situación económica en los casos en los que es baja y en los que es lo contrario, aumentar la atención a los menores y no descuidarlos. Los menores deben sentir seguridad en todos los sentidos: Social, emocional y mental. Puede ser tratado por diferentes intervenciones, ya sean, educativas, clínicas, familiares, sociales, entre otras. Los programas de prevención social de la violencia tienen por objetivo prevenir la conducta 
antisocial en la población infantil-juvenil, mediante la implementación de estrategias y acciones dirigidas a la reorientación de los menores.

\section{REFERENCIAS BIBLIOGRÁFICAS}

Alessandroni, N. (2017). Imaginación, creatividad y fantasía en Lev. S. Vygotski: una aproximación a su enfoque sociocultural. Actualidad en Psicología, Vol. 31, p.p. 45 - 60. https://www.scielo.sa.cr/pdf/ap/v31n122/2215-3535-ap-31-122-00045.pdf

American Psychiatric Association (2014). Manual Diagnóstico y Estadístico de los Trastornos Mentales. DSM-5. Ciudad de México: Editorial Médica Panamericana.

Bayo, J. (1987). Estudio sobre el desarrollo de la percepción visual en el niño. Análisis de la exploración visual de la pintura moderna por una muestra infantil. Barcelona: Universitat de Barcelona. https://dialnet.unirioja.es/servlet/tesis?codigo=135160

Bayón, F. (2005). Juventud y prisión. Revista de Estudios de Juventud, Vol. 69, p.p. 9-29. http://www.injuve.es/sites/default/files/revista69completa.pdf

Becoña, E. (2000). Los adolescentes y el consumo de drogas. Papeles del Psicólogo, Vol. 77, p.p. 25 - 32. https://www.redalyc.org/pdf/778/77807705.pdf

Bizberg, I. (2015). México: Una transición fallida. Desacatos, Vol. 48, p.p. 122 - 139. https://www.redalyc.org/pdf/139/13938788009.pdf

Calderón, D. (2015). Los niños como sujetos sociales. Notas sobre la antropología de la infancia. Nueva Antropología, Vol. 28, p.p. 125 - 140. http://www.scielo.org.mx/pdf/na/v28n82/v28n82a7.pdf

Cámara de Diputados (2019). Ley Federal contra la Delincuencia Organizada. Ciudad de México: Cámara de Diputados. http://www.diputados.gob.mx/LeyesBiblio/pdf/101_081119.pdf

Cámara de Diputados (2016). Ley Nacional del Sistema Integral de Justicia Penal para Adolescentes. Ciudad de México: Cámara de Diputados. http://www.diputados.gob.mx/LeyesBiblio/pdf/LNSIJPA.pdf

Castillejos, D. (2011). Análisis constitucional sobre el uso del término menor, y los de niños, niñas y adolescentes. Publicación Electrónica, No. 5. Biblioteca Jurídica Virtual de la UNAM. https://archivos.juridicas.unam.mx/www/bjv/libros/7/3011/10.pdf 
Wael Sarwat Hikal Carreón

Comisión Interamericana de Derechos Humanos (2015). Violencia, Niñez y Crimen Organizado. Washington: Organización de los Estados Americanos. Recuperado de http://www.oas.org/es/cidh/informes/pdfs/ViolenciaNinez2016.pdf

Comisión Nacional de los Derechos Humanos, Universidad Nacional Autónoma de México y Coordinación de Humanidades (2019). Niñas, Niños y Adolescentes Víctima del Crimen Organizado en México. Ciudad de México: Comisión Nacional de los Derechos Humanos. https://www.cndh.org.mx/sites/default/files/documentos/2019-11/Estudio-ninas-ninosadolescentes-victimas-crimen.pdf

Comisión Nacional de los Derechos Humanos (2018). Ley General de los Derechos de Niñas, Niños y Adolescentes. Ley General de Prestación de Servicios para la Atención, Cuidado y Desarrollo Integral Infantil. Ciudad de México: Comisión Nacional de los Derechos Humanos. https://www.cndh.org.mx/sites/default/files/doc/Programas/Ninez_familia/Material/leyguarderias-ninos.pdf

Conde, E. (2015). Empatía y conducta antisocial en adolescentes: convergencia de dos medidas de empatía y su relación con la conducta antisocial. Facultad de Derecho, Centro de Investigación en Criminología, Universidad de Castilla-La Mancha. https://ruidera.uclm.es/xmlui/bitstream/handle/10578/10014/TESIS\%20Conde\%20Alvara do.pdf?sequence $=1 \&$ is Allowed $=\mathrm{y}$

Cornellá, J. y Llusent, Á. (Sin Fecha). Agresividad y violencia en el niño y en el adolescente. Sociedad Española de Pediatría Extrahospitalaria y Atención Primaria. https://sepeap.org/wpcontent/uploads/2014/02/Ps_inf_agresividad_violencia.pdf

Dávila, Ó. (2004). Adolescencia y juventud: De las nociones a los abordajes. Ultima década, Vol.12, p.p. 83 - 104. https://scielo.conicyt.cl/pdf/udecada/v12n21/art04.pdf

Escobar, G. (2006). La enseñanza en criminología. Algunas consideraciones. Revista Electrónica de Ciencia Penal y $\quad$ Criminología. $\quad 1 \quad-\quad 8$. http://criminet.ugr.es/recpc/08/recpc08-r2.pdf

Flores, R. (10 de septiembre de 2019). Adolescentes, los sicarios desechables del Narco. Excelsior. https://www.excelsior.com.mx/nacional/adolescentes-los-sicarios-desechables$\underline{\text { del-narco/1335471 }}$ 
Hernández, L. (2011). Salus. Adolescencia: ¿Adolecer es padecer? Vol. 15, No. 2, p.p. 5 8. http://ve.scielo.org/pdf/s/v15n2/art03.pdf

Horacio, L. (2015). “Ganarse el respeto". Un análisis de los conflictos de la sociabilidad juvenil en la escuela secundaria. Revista Mexicana de Investigación Educativa, 67(20), 1105-1130. Recuperado de https://www.redalyc.org/pdf/140/14042022005.pdf

Infobase (2019). Joselyn, "la niña sicaria" que apareció descuartizada en una hielera. https://www.infobae.com/america/mexico/2019/09/06/joselyn-la-nina-sicaria-queaparecio-descuartizada-en-una-hielera/

Infobase (2019). "Juanito Pistolas": el sicario de 16 años que tenía su propio rap y murió decapitado en Nuevo Laredo. https://www.infobae.com/america/mexico/2019/09/07/asesinaron-a-presunto-sicario-enrio-verde-tenia-antecedentes-criminales-desde-los-10-anos/

Infobase (2019). Asesinaron a presunto sicario en Rio Verde: tenía antecedentes criminales desde los 10 años. https://www.infobae.com/america/mexico/2019/08/28/juanito-pistolasel-sicario-de-16-anos-que-tenia-su-propio-rap-y-murio-decapitado-en-nuevo-laredo/

Infobase (2019). Niño "narco", que tiene de mascota a un tigre, amenazó a rivales del "Mayo" Zambada. https://www.infobae.com/america/mexico/2019/08/31/nino-narco-quetiene-de-mascota-a-un-tigre-amenazo-a-rivales-del-mayo-zambada/

Informador (5 de noviembre 2019). Adolescentes sicarios revelan que los atraen vida de lujos y narcocultura. Informador. https://www.informador.mx/mexico/Adolescentessicarios-revelan-que-los-atraen-vida-de-lujos-y-narcocultura-20191105-0067.html

Institute for Economics \& Peace (2018). Global Peace Index 2019. Sydney: Institute for Economics \& Peace. http://visionofhumanity.org/indexes/global-peace-index/

Instituto Nacional de Estadística y Geografía (2016). Población reclusa y adolescentes infractores. https://www.inegi.org.mx/temas/poblacion/

Jaramillo, L. (2007). Concepciones de infancia. Zona Próxima, Vol. 8, p.p. 108 - 123. https://www.redalyc.org/pdf/853/85300809.pdf

Job, V. (2019). Inegi: en el país, 5 mil 888 niños bajo proceso o con sentencia. Revista Milenio. https://www.milenio.com/policia/inegi-5-mil-888-ninos-proceso-sentencia 
Wael Sarwat Hikal Carreón

Lanadown, G. (2005). La Evolución de las Facultades del Niño. Florencia: Fondo de las Naciones Unidas para la Infancia. https://www.unicefirc.org/publications/pdf/EVOLVING-E.pdf

La Redacción (30 de julio de 2013). Más de 121 mil muertos, el saldo de la narcoguerra de Calderón: Inegi. Blog Proceso. https://www.proceso.com.mx/348816/mas-de-121-milmuertos-el-saldo-de-la-narcoguerra-de-calderon-inegi

Lara, I. (5 de diciembre de 2018). Hubo más ejecuciones con Enrique Peña Nieto que con Felipe Calderón. Blog Proceso. https://www.proceso.com.mx/562597/hubo-masejecuciones-con-enrique-pena-nieto-que-con-felipe-calderon

Manrique, M. (2013). Ignorancia deliberada y responsabilidad penal. Isonomía, Vol. 40, p.p. 163 - 195. http://www.scielo.org.mx/pdf/is/n40/n40a8.pdf

Martínez, B.; Méndez, E.; y Pérez, E. (2014). Expectativas de vida, género y ruralidad de jóvenes en una comunidad migrante del estado de Puebla, México, Agricultura, Sociedad y Desarrollo, Vol. 3, No. 11, p.p. $337 \quad$ - 357. http://www.scielo.org.mx/pdf/asd/v11n3/v11n3a5.pdf

Martínez, M.; Robles, C.; Utria, L.; y Amar, J. (2014). Legitimación de la violencia en la infancia: Un abordaje desde el enfoque ecológico de Bronfenbrenner, Psicología desde el Caribe, Vol. 1, No. 31, p.p. 133 - 160. https://www.redalyc.org/pdf/213/21330429007.pdf

Martínez, A. (2016). La violencia. Conceptualización y elementos para su estudio. Política y Cultura, Vol. 46, p.p. 7 - 31. https://www.redalyc.org/pdf/267/26748302002.pdf

México SOS (Sin Fecha). Jóvenes: Entre la delincuencia y la agresión. Recuperado de http://www.mexicosos.org/dossier/librero-sos/870-jovenes-entre-la-delincuencia-y-la$\underline{\text { agresion }}$

Mendoza, J. (2011). La tortura en el marco de la guerra sucia en México: un ejercicio de memoria colectiva. Polis: Investigación y Análisis Sociopolítico y Psicosocial, Vol. 7, No. 2 , p.p. 139 179. https://www.redalyc.org/pdf/726/Resumenes/Resumen_72621412006_1.pdf

Mustaca, A. (2018). Frustración y conductas sociales. Avances en Psicología Latinoamericana, $\quad$ Vol. $\quad 36, \quad$ No. $\quad 1, \quad$ p.p. $\quad 65 \quad$ - 81. http://www.scielo.org.co/pdf/apl/v36n1/1794-4724-apl-36-01-00065.pdf 
Ochoa Rojas, A.C. (2007). Análisis de la percepción infantil con respecto a la familia a partir de la caricatura "Franklin y sus amigos". Investigación Universitaria Multidisciplinaria: Revista de Investigación de la Universidad Simón Bolívar, Vol. 6, p.p. 35-47. https://dialnet.unirioja.es/descarga/articulo/2695341.pdf

Oficina de las Naciones Unidas contra la Droga y el Delito (2020). Crimen Organizado Transnacional. S.P: Oficina de las Naciones Unidas contra la Droga y el Delito. https://www.unodc.org/ropan/es/organized-crime.html

Oficina de las Naciones Unidas contra la Droga y el Delito (2007). Recopilación de Reglas y Normas de las Naciones Unidas en la Esfera de la Prevención del Delito y la Justicia Penal.

https://www.unodc.org/pdf/criminal_justice/Compendium UN Standards and Norms C $\underline{P \_ \text {and_CJ_Spanish.pdf }}$

Organización Mundial de la Salud (2020). Desarrollo en la adolescencia. https://www.who.int/maternal child adolescent/topics/adolescence/dev/es/

Ortega, R. \& Somuani, M. (2015). El periodo presidencial de Felipe Calderón Hinojosa. Foro Internacional, Vol. 55, No. $\quad$ 1, $\quad$ p.p. $\quad$ 5-15. http://www.scielo.org.mx/pdf/fi/v55n1/0185-013X-fi-55-01-00005.pdf

Pérez-Stadelmann, C. (2015). Teníamos ganas de matar a Christopher, El Universal. https://www.eluniversal.com.mx/articulo/nacion/sociedad/2015/08/23/teniamos-ganas-dematar-christopher

Puértolas, M. (7 de noviembre de 2019). Hoy sicarios matan niños. Revista Milenio. https://www.milenio.com/opinion/miguel-angel-puertolas/en-frecuencia/hoy-sicarios$\underline{\text { matan-ninos }}$

Raffo, H. (2000). Menores Infractores y Libertad Asistida. Buenos Aires, Ediciones La Rocca.

Ramírez, C. (19 de diciembre de 2009). Delincuencia juvenil. Psicoanálisis, Cultura, Arte y Tecnología. Blog "Entre broma y broma la verdad se asoma" http://camiloramirezgarza.blogspot.com/2009/

Rivera, C. (1 de mayo de 2017). En las filas del crimen, al menos 30 mil niños. Revista Milenio. https://www.milenio.com/policia/en-las-filas-del-crimen-al-menos-30-mil-ninos 
Wael Sarwat Hikal Carreón

Romero, I. (2004). Desvelar la violencia: Una intervención para la prevención y el cambio. Papeles del Psicólogo, Vol. 25, No. $\quad 88, \quad 19 \quad$ - 25. https://www.redalyc.org/pdf/778/77808803.pdf

Sánchez, A. (2017). Historia del penitenciarismo en México. Biblioteca Jurídica Virtual del Instituto de Investigaciones Jurídicas de la UNAM. https://archivos.juridicas.unam.mx/www/bjv/libros/10/4770/29.pdf

Statistics Iceland (2019). The population increased by 1,610 in the second quarter of 2019. https://www.statice.is/publications/news-archive/inhabitants/population-in-the-2ndquarter-2019/

Secretariado Ejecutivo del Sistema Nacional de Seguridad Pública (2019). Incidencia delictiva. https://www.gob.mx/sesnsp/acciones-y-programas/incidencia-delictiva-87005

Senado de la República (2014). Situación actual y factores que influyen en la delincuencia juvenil. Mirada Legislativa, 51. http://www.bibliodigitalibd.senado.gob.mx/bitstream/handle/123456789/1993/ML51.pdf? sequence $=1 \&$ is Allowed $=\mathrm{y}$

Suárez Beltrán, M.L. (2009). La experiencia de la imaginación creadora como elemento primordial de la creación poética en la infancia. Civilizar, Vol. 9, No. 17, p.p. 169 - 179.

Tieghi, O.N. (2010). Acerca de la edad de inimputabilidad en Argentina. Archivos de Criminología, Criminalística y Seguridad Privada, 4, 1 - 7.

Torres, C. (2005). Jóvenes y violencia. Revista Iberoamericana de Educación, Vol. 37. Recuperado de https://rieoei.org/historico/documentos/rie37a03.htm

UNICEF Comité Español (2006). Convención sobre los Derechos del Niño. Madrid: UNICEF Comité Español. Recuperado de https://www.un.org/es/events/childrenday/pdf/derechos.pdf

Velázquez, I. (2005). Una aproximación al mapa disciplinar de la pedagogía. Revista Iberoamericana de Educación, Vol. 35, No. 2, p.p. 1 - 15.

Zuckermann, L. (2019). ¿Los muertos de AMLO? Excelsior. Recuperado de https://www.excelsior.com.mx/opinion/leo-zuckermann/los-muertos-de-amlo/1350994 